$\mathrm{DOI}$

\title{
CLINICAL AND FUNCTIONAL CHARACTERISTICS OF RESISTANT ARTERIAL HYPERTENSION
}

\section{Odesa National Medical University}

SUMMARY. The aim of the work - to study clinical and functional characteristics of resistant and pseudo-resistant arterial hypertension.

Materials and Methods. 420 patients with essential arterial hypertension of $2^{\text {nd }}$ stage at the age from 45 to 74 who were ill for more than 3 years with signs of resistance to hypertensive therapy were examined. All patients were divided into 2 groups: the group 1 with resistant AH and the group 2 with pseudo-resistant AH. All patients' data about anamnesis, complaints, duration of being ill, existence of concomitant disease was estimated. All necessary general clinical and instrumental examinations were made according to set tasks of research: general clinical examination, ECG, EchoCG.

Results. During the research period in $26.2 \%$ of patients we diagnosed true resistant $\mathrm{AH}, 73.8 \%$ - pseudoresistant $A H$. Duration of pseudo-resistant $A H$ averagely reached (8.2 \pm 1.2 ) years, and of resistant $A H-a l m o s t ~ t w o$

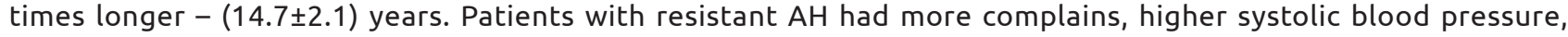
more frequently had comorbidity and heart rhythm and conduction disorder in comparison with patients with pseudo-resistant $\mathrm{AH}$.

Conclusions. Consequently more difficult $\mathrm{AH}$ progression is registered within patients with resistant $\mathrm{AH}$, what creates conditions for increase of general cardiovascular risk.

KEY WORDS: resistant arterial hypertension; pseudo-resistant arterial hypertension; clinical and functional characteristics.

Introduction. Resistant arterial hypertension (RAH) is one of the most significant modifiable risk factors of cardiovascular morbidity and mortality. RAH stimulates development of such life-threatening and incapacitating complications as miocardial infarction, sharp left ventricular and kidney failure, hemorrhagic stroke, vision disorders $[1,2,3,4,5]$. In spite of perfection of arterial hypertension (AH) diagnostic methods, existence of broad spectrum of antihypertensive medications, therapy-resistant arterial hypertension (AH) stay an unsettled clinical problem with which both general practice doctors and doctors of other specialties (cardiologists, neurologists) have to deal $[6,7,8]$. According to WHO data only $50 \%$ of patients who are under medical treatment of antihypertensive medication, manage to react $A B P$ control $[2,3]$. According to epidemiological survey data in Ukraine, less than $19 \%$ of patients in cities and only $8 \%$ - in rural localities reach lover ABP level than $140 / 90 \mathrm{~mm}$ Mercury $[9,10]$. Literature data, devoted to study of refractory mechanisms emergence are rare and fragmentary, what justifies carrying out a more in-depth study of the issue.

Significant quantity of patients' data is falsely classified as those who have resistant $\mathrm{AH}$. This diagnosis does not always correspond to reality and is often falsely made to patients with pseudo-resistant AH. During examination of such patients a sound exclusion of potential reasons of pseudo-resistance should be made, removal of which provokes a necessary decrease of ABP. Pseudo-resistant AH is driven mainly by low adherence to medicinal and non-medicinal treatment, irrational assignment regimen (inadequacy of doses and com- binations) of antihypertensive medication, and also by inobservance of a healthy lifestyle (continue smoking, abusive drinking behavior, food with great amount of salt and so on) $[2,6,7,9]$.

Consequently, it seems urgent to continue researches directed for further research of clinical and functional features of resistant arterial hypertension, for development of the most efficient and affordable patient examination algorithms with the aim of maximum early detection of true arterial hypertension, reasons of development of pseudo-resistance and their correction at stage of a primary link of medical assistance.

The aim of the study - to study clinical and functional characteristics of resistant and pseudo-resistant arterial hypertension.

Materials and Methods. 420 patients with essential arterial hypertension of 2 stage at the age from 45 to 74 who were ill for more than 3 years with signs of resistance to antihypertensive therapy were exterminated. All patients were divided into 2 groups: the group 1 - patients with resistant $\mathrm{AH}$ and the group 2 - patients with pseudo-resistant $\mathrm{AH}$. Differential exclusion of true resistant $\mathrm{AH}$ and pseudo-resistant was carried out according to AHA recommendations.

All patients' data of anamnesis, complaints, duration of being $\mathrm{AH}$ ill, existence of concomitant disease was estimated. Following examinations were made to all patients: general clinical examination, examination complex included measurement of ABP by Korotkov method according to ESH/ESC recommendations in 12 standard deviations, EchoCG. 
Огляди літератури, оригінальні дослідження, погляд на проблему

Statistical processing of the research results was made with use of common methods of variation statistics with the help of Microsoft Excel 7 program packs using Student's t-test.

Results and Discussions. According to the results of the research $26.6 \%$ of patients were diagnosed true resistant $A H$, and $73.8 \%$ - pseudo-resistant AH (Pict. 1).

Average duration of disease among exterminated patients with pseudo-resistant $A H$ was $8.2 \pm 1.2$ years, and of resistant $\mathrm{AH}$ - almost two times longer $14.7 \pm 2.1$ years.

Comparative analysis of clinical and anamnestic data of both groups shoved that patients with resis- tant $\mathrm{AH}$ had more much more complaints than patients with pseudo-resistant AH (Tab.1). Particularly, complaints for headache $(p<0.001)$, faintness $(p<0.01)$, heartache $(p<0.001)$, distribution of sleep $(p<0.001)$, undue fatiguability $(p<0.001)$, exertional breathlessness $(p<0.001)$ were marked more often.

Patients with essential resistant AH had comorbidity more often than patients with pseudo-resistant, but it was not the reason for elevated blood pressure emergence (Tab. 2). So, patients with resistant $\mathrm{AH}$ had diseases with pathogenic features more often than patients with pseudo-resistant $\mathrm{AH}$, namely: ischemic heart disease $(<0.001)$, chronic cardiac insufficiency of 1 and 2 stages ( $<0.001)$.

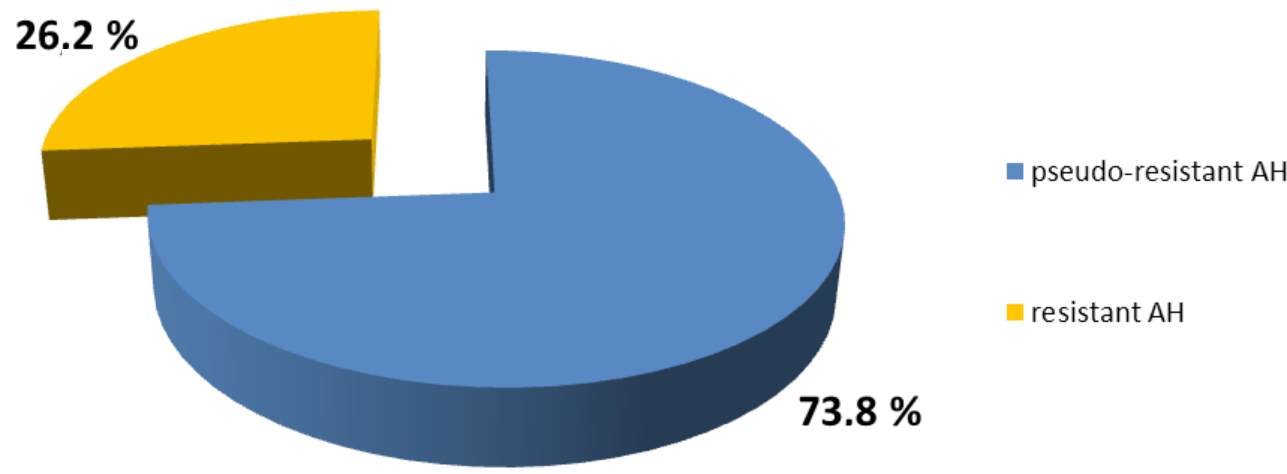

Picture 1. Structure of patients with essential arterial hypertension of 2 stage with signs of resistance to antihypertensive therapy.

Table 1. Main complaints of exterminated patients ( $\mathrm{P} \pm \mathrm{q}, \%)$

\begin{tabular}{|l|c|c|c|}
\hline \multicolumn{1}{|c|}{ Complaints } & Pseudo-resistant AH & Resistant AH & P \\
\hline Frequent headache & $48.1 \pm 2.8$ & $61.8 \pm 4.6$ & $<0.001$ \\
\hline Faintness & $16.1 \pm 2.1$ & $33.6 \pm 4.5$ & $<0.01$ \\
\hline Heartache & $38.1 \pm 2.8$ & $64.5 \pm 4.6$ & $<0.001$ \\
\hline Distribution of sleep & $57.1 \pm 2.8$ & $78.2 \pm 3.9$ & $<0.001$ \\
\hline $\begin{array}{l}\text { Exertional } \\
\text { breathlessness }\end{array}$ & $39.0 \pm 2.8$ & $68.2 \pm 4.4$ & $<0.001$ \\
\hline Undue fatiguability & $57.1 \pm 2.8$ & $91.8 \pm 2.6$ & $<0.001$ \\
\hline Tachycardia strokes & $23.9 \pm 2.4$ & $25.5 \pm 4.2$ & $<0.5$ \\
\hline Heart rhythm disturbance & $15.9 \pm 2.0$ & $25.5 \pm 4.2$ & $<0.3$ \\
\hline
\end{tabular}

Table 2. Frequency of comorbidity of exterminated patients $(P \pm q, \%)$

\begin{tabular}{|l|c|c|c|}
\hline \multicolumn{1}{|c|}{ Diseases } & Pseudo-resistant AH & Resistant AH & P \\
\hline Ischemic heart disease & $11.9 \pm 1.8$ & $31.8 \pm 4.4$ & $<0.001$ \\
\hline Chronic cardiac insufficiency of 1 stage & $71.9 \pm 2.6$ & $46.4 \pm 4.8$ & $<0.001$ \\
\hline Chronic cardiac insufficiency of 2 stage & $28.1 \pm 2.6$ & $53.6 \pm 4.8$ & $<0.001$ \\
\hline Chronic obstructive lung disease & $24.5 \pm 2.4$ & $28.2 \pm 4.3$ & $<0.5$ \\
\hline Diabetes mellitus & $15.2 \pm 2.0$ & $60.0 \pm 4.7$ & $<0,001$ \\
\hline Cholecystitis & $21.9 \pm 2.3$ & $25.5 \pm 4.2$ & $<0.3$ \\
\hline Pancreatitis & $24.5 \pm 2.4$ & $30.0 \pm 4.4$ & $<0.3$ \\
\hline Arthrosis & $40.0 \pm 2.8$ & $30.0 \pm 4.4$ & $<0.1$ \\
\hline Radiculitis & $80.0 \pm 2.8$ & $70.0 \pm 4.4$ & $<0.1$ \\
\hline
\end{tabular}


Огляди літератури, оригінальні дослідження, погляд на проблему

Data of analysis of hemodynamic parameters showed that patients with resistant AH had higher level of intraday average, daily and nightly systolic blood pressure, higher level of nightly diastolic blood pressure than patients with pseudo-resistant $\mathrm{AH}$. Also group of patients with resistant $\mathrm{AH}$ had higher variability of systolic blood pressure during twenty-four hours and in the night time than patients with pseudo-resistant AH (Tab.3).
According to data of instrumental diagnostics (electrocardiography and echocardiography) the group with resistant AH had the biggest percent of cases of hypertrophy of interventricular septum, myocardial mass (men) more than $150 \mathrm{~g}$ and myocardial mass (women) more than $130 \mathrm{~g}$, ECG - indications of left atrial enlargement (Tab.4). Received data proves results of multiple researches relative to resistant $\mathrm{AH}[2,3,4]$.

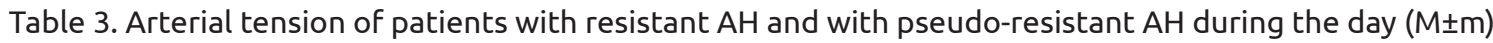

\begin{tabular}{|c|c|c|c|}
\hline Tension & Pseudo-resistant $\mathrm{AH}$ & Resistant $\mathrm{AH}$ & $\mathrm{P}$ \\
\hline \multicolumn{4}{|c|}{ twenty-four hours } \\
\hline $\begin{array}{l}\text { Intraday average systolic blood pressure, } \\
\text { mm Mercury }\end{array}$ & $142.2 \pm 2.4$ & $149.7 \pm 2.2$ & $<0.05$ \\
\hline $\begin{array}{l}\text { Intraday average diastolic blood pressure, } \\
\text { mm Mercury }\end{array}$ & $88.1 \pm 2.0$ & $92.9 \pm 1.7$ & $>0.1$ \\
\hline $\begin{array}{l}\text { Std. dev. systolic blood pressure, mm } \\
\text { Mercury }\end{array}$ & $22.1 \pm 1.1$ & $32.9 \pm 0.8$ & $<0.001$ \\
\hline $\begin{array}{l}\text { Std. dev. diastolic blood pressure, mm } \\
\text { Mercury }\end{array}$ & $12.9 \pm 2.4$ & $16.9 \pm 0.7$ & $>0.5$ \\
\hline \multicolumn{4}{|c|}{ Day } \\
\hline $\begin{array}{l}\text { Daily average systolic blood pressure, mm } \\
\text { Mercury }\end{array}$ & $141.7 \pm 3.3$ & $149.3 \pm 2.2$ & $<0.05$ \\
\hline Daily average diastolic & $78.2 \pm 2.2$ & $80.9 \pm 1.5$ & $>0.5$ \\
\hline \multicolumn{4}{|l|}{ blood pressure, mm Mercury } \\
\hline $\begin{array}{l}\text { Std. dev. systolic blood pressure, mm } \\
\text { Mercury }\end{array}$ & $23.1 \pm 1.3$ & $39.1 \pm 0.9$ & $<0.001$ \\
\hline $\begin{array}{l}\text { Std. dev. diastolic blood pressure, mm } \\
\text { Mercury }\end{array}$ & $12.2 \pm 2.2$ & $13.9 \pm 0.7$ & $>0.5$ \\
\hline \multicolumn{4}{|c|}{ Night } \\
\hline $\begin{array}{l}\text { Nightly average systolic blood pressure, } \\
\text { mm Mercury }\end{array}$ & $140.3 \pm 3.1$ & $148.3 \pm 2.2$ & $<0.05$ \\
\hline $\begin{array}{l}\text { Nightly average diastolic blood pressure, } \\
\text { mm Mercury }\end{array}$ & $68.1 \pm 2.0$ & $76.9 \pm 1.5$ & $<0.01$ \\
\hline $\begin{array}{l}\text { Std. dev. systolic blood pressure, mm } \\
\text { Mercury }\end{array}$ & $18.1 \pm 1.1$ & $23.3 \pm 1.2$ & $<0.05$ \\
\hline $\begin{array}{l}\text { Std. dev. diastolic blood pressure, mm } \\
\text { Mercury }\end{array}$ & $14.9 \pm 2.4$ & $13.7 \pm 1.1$ & $>0.5$ \\
\hline
\end{tabular}

Table 4. Frequency and nature of change of systemic (left) heart according to patients' data of electrocardiography and echocardiography, $\mathrm{P} \pm \mathrm{q}, \%$

\begin{tabular}{|l|c|c|c|}
\hline \multicolumn{1}{|c|}{ Indices } & Pseudo-resistant AH & Resistant AH & P \\
\hline Hypertrophy of back wall of the left ventricle & 100.0 & 100.0 & $<0.001$ \\
\hline Hypertrophy of interventricular septum & $78.2 \pm 3.9$ & $99.1 \pm 0.9$ & $>0.05$ \\
\hline EF less than $50 \%$ & $11.8 \pm 3.1$ & $19.1 \pm 3.7$ & $<0.01$ \\
\hline Myocardial mass (men) more than $150 \mathrm{~g}$ & $78.2 \pm 3.9$ & $89.1 \pm 3.0$ & $<0.001$ \\
\hline Myocardial mass (women) more than 130 g & $65.5 \pm 4.5$ & $95.5 \pm 0.7$ & $<0.001$ \\
\hline Diastolic dysfunction & $88.2 \pm 3.1$ & $81.8 \pm 3.7$ & $>0.2$ \\
\hline Sokolov-Lyons index more than 35 mm & $88.1 \pm 1.8$ & 100.0 & - \\
\hline $\begin{array}{l}\text { ECG - indications of left ventricle myocardial } \\
\text { hypertrophy }\end{array}$ & 100.0 & $69.1 \pm 4.4$ & $<0.001$ \\
\hline ECG - indications of left atrial enlargement & $41.9 \pm 2.8$ & & \\
\hline
\end{tabular}


Огляди літератури, оригінальні дослідження, погляд на проблему

Analysis of ECG data showed that patients with resistant $\mathrm{AH}$ have heart rhythm and conduction dis- order more often than patients with pseudo-resistant AH (Pict. 2).

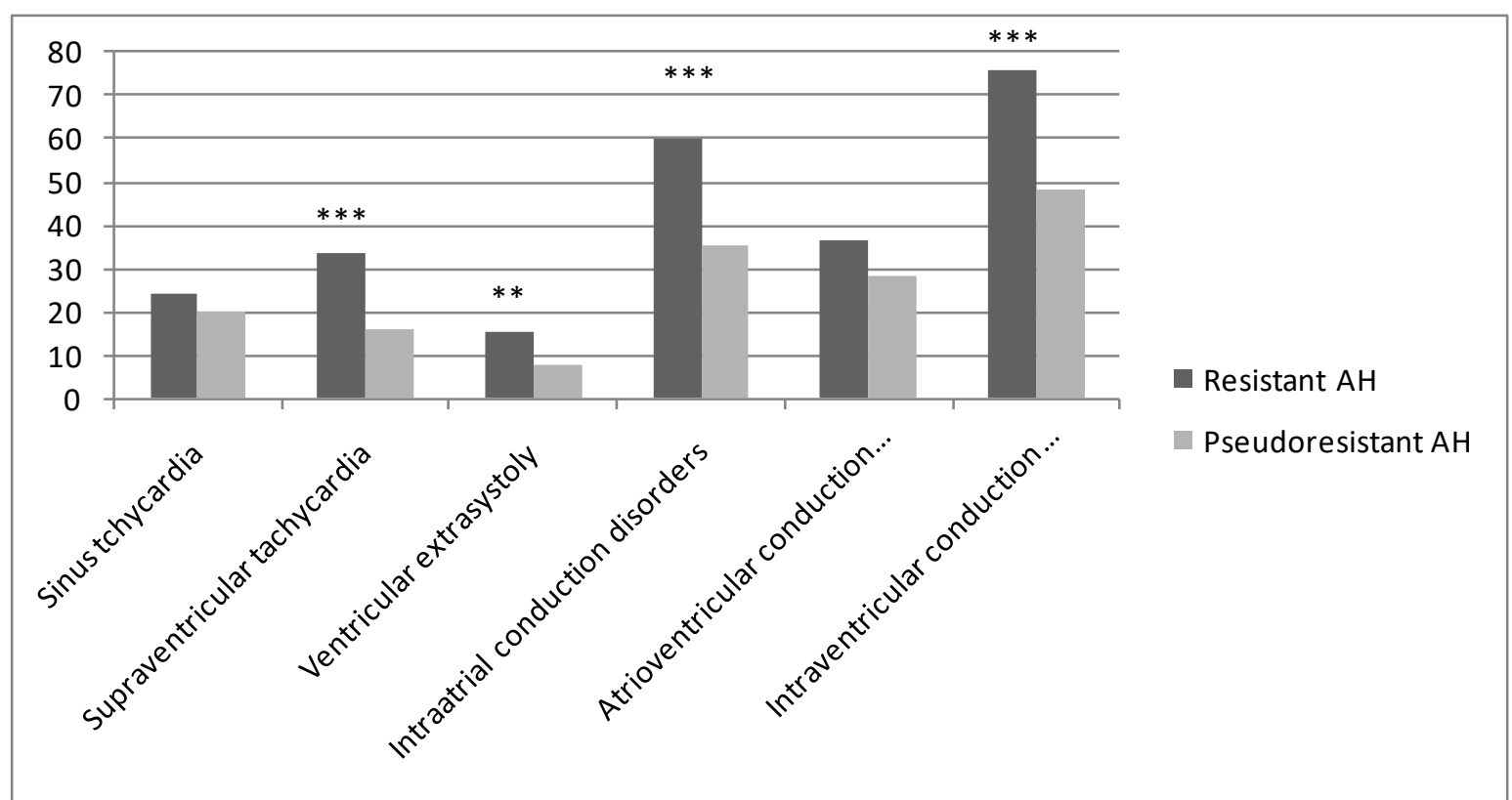

Picture 2. Frequency of rhythm disturbances and conduction disorders in examined patients. NB: ** - significant differences between groups $(p<0.01), * * *-$ significant differences between groups $(p<0.001)$

Conclusions. The carried out research proved that patients with resistant $\mathrm{AH}$ have more difficult $\mathrm{AH}$ course. Majority of patients with resistant $\mathrm{AH}$ have comorbidities: Ischemic heart disease, chronic cardiac insufficiency, diabetes mellitus, which make disease state more difficult. Patients with resistant arterial hypertension showed higher frequency of cardiac rhythm and conduction disorders.

\section{ЛІТЕРАТУРА}

1. Guidelines for the management of arterial hypertension: The Task Force for the management of arterial hypertension of the European Society of Hypertension (ESH) and European Society of Cardiology (ESC) / G. Mancia, R. Fagard, K. Narkiewicz [et al.] // J. Hypertens. - 2013. - № 31. - P. 1281-1357.

2. David A. C. Resistant Hypertension: Diagnosis, Evaluation, and Treatment: A Scientific Statement From the American Heart Association Professional Education Committee of the Council for High Blood Pressure Research / A. C. David, J. Daniel, T. Stephen // Hypertension. - 2008. - P. 1403-1419.

3. Pimenta E. Resistant Hypertension: Incidence, Prevalence, and Prognosis / E. Pimenta, D. A. Calhoun // Circulation. - 2012. - № 125 (13) - P. 1594-1596.

4. Worldwide prevalence of hypertension: a systematic review / P. Kearney, M. Whelton, K. Reynolds [et al.] // J. of Hypertens. - 2004. - № 22. - P. 11-19.

5. Hypertension prevalence and blood pressure levels in European countries, Canada and the United States / K. Wolf-Maier, R. S. Cooper, J. R. Banegas [et al.] // JAMA. 2003. - № 289. - P. 2363-2369.
6. Sirenko Y. M. Hypertension and comorbidity / Sirenko Y. M. - Donetsk : Publisher O. Zaslavsky, 2010.

7. Deneka I. E. Resistant hypertension: epidemiology and risk factors / I. E. Deneka, A. A. Svetankova, A. V. Rodionov // Rational pharmacotherapy in kardiology. - 2016. - № 12 (4). - P. 459-464.

8. Mathers C. Global health risks: mortality and burden of disease attributable to selected major risks / C. Mathers, G. Stevens, M. Mascarenhas - Geneva, Switzerland: World Health Organization, 2009.

9. Obertynska O. G. Clinical aspects of resistant hypertension / O. G. Obertynska // Ukr. Card. journal. -2014. - № 4. -P. 30-36.

10. Obertynska O. G. Resistant hypertension: search for optimal combination therapy / O. G. Obertynska // Ukrainian Cardiology journal. - 2015. - № 6. - № 113-123.

11. Resistant Hypertension: Diagnosis, Evaluation, and Treatment. A Scientific Statement From the American Heart Association Professional Education Committee of the Council for High Blood Pressure Research // Hypertension. - 2008. - № 51. - P. 1403-1419. 
Огляди літератури, оригінальні дослідження, погляд на проблему

REFERENCES

1. Mancia, G., Fagard, R., \& Narkiewicz, K. (2013). Guidelines for the management of arterial hypertension: The Task Force for the management of arterial hypertension of the European Society of Hypertension (ESH) and European Society of Cardiology (ESC). J. Hypertens. 31, 1281-1357.

2. David, A. C., Daniel, J., \& Stephen, T. (2008). Resistant hypertension: diagnosis, evaluation, and treatment: a scientific statement from the American Heart Association Professional Education Committee of the Council for High Blood Pressure Research. Hypertension, 1403-1419.

3. Pimenta, E., \& Calhoun, D.A. (2012) Resistant Hypertension: Incidence, Prevalence, and Prognosis. Circulation, 125 (13), 1594-1596.

4. Kearney, P., Whelton, M., \& Reynolds, K. (2004). Worldwide prevalence of hypertension: a systematic review. J. of Hypertens., 22, 11-19.

5. Wolf-Maier, K., Cooper, R. S., \& Banegas, J. R. (2003). Hypertension prevalence and blood pressure levels in European countries, Canada and the United States. JAMA. 289, 2363-2369.
6. Sirenko, Y.M. (2010). Hypertension and comorbidity. Donetsk: Publisher O. Zaslavsky.

7. Deneka, I.E., Svetankova, A.A., \& Rodionov, A.V. (2016). Resistant hypertension: epidemiology and risk factors. Rational Pharmacotherapy in Cardiology, 12 (4), 459-464.

8. Mathers, C., Stevens, G., \& Mascarenhas, M. (2009). Global health risks: mortality and burden of disease attributable to selected major risks. Geneva, Switzerland: World Health Organization.

9. Obertynska, O.G. (2014). Clinical aspects of resistant hypertension. Ukr. Card. Journal, 4, 30-36.

10. Obertynska, O.G. (2015). Resistant hypertension: search for optimal combination therapy. Ukrainian Cardiology Journal, 6, 113-123.

11. Resistant hypertension: diagnosis, evaluation, and treatment. a scientific statement from the American Heart Association Professional Education Committee of the Council for High Blood Pressure Research (2008). Hypertension, 51, 1403-1419.

\section{КЛІНІКО-ФУНКЦІОНАЛЬНА ХАРАКТЕРИСТИКА РЕЗИСТЕНТНОСТІ АРТЕРІАЛЬНОЇ ГІПЕРТЕНЗІї}

○С. В. Бусел

\section{Одеський начіональний медичний університет}

РЕзЮМЕ. Мета дослідження - вивчити клініко-функціональні особливості резистентної і псевдорезистентної артеріальної гіпертензії.

Матеріали і методи. Було обстежено 420 хворих з есенціальною артеріальною гіпертензією 2 стадії у віці від 45 до 74 років з тривалістю захворювання понад 3 роки з ознаками резистентності до проведеної гіпотензивної терапії. Всі пацієнти були поділені на 2 групи: 1 група - з резистентною АГ і 2 група - з псевдорезистентною АГ. У всіх пацієнтів оцінювали дані анамнезу, скарги, тривалість захворювання АГ, наявність супутніх захворювань. Були проведені всі необхідні загальноклінічні та інструментальні обстеження відповідно до поставлених завдань дослідження: загальноклінічне дослідження, ЕКГ, ЕхоКГ.

Результати. В ході дослідження у 26,2 \% пацієнтів була діагностована справжня резистентна АГ, у 73,8 \% псевдорезистентна АГ. Тривалість псевдорезистентної АГ в середньому склала $(8,2 \pm 1,2)$ років, а резистентної АГ - майже вдвічі більше - $(14,7 \pm 2,1)$ років. У хворих з резистентною АГ, порівняно з хворими з псевдорезистентною АГ, було виявлено більше скарг, вищі значення систолічного артеріального тиску, частіше зустрічалися супутня патологія і порушення серцевого ритму і провідності.

Висновки. У хворих з резистентною АГ відзначається більш тяжкий перебіг АГ, що створює умови для підвищення загального кардіоваскулярного ризику.

КЛючОВІ СЛОВА: резистентна артеріальна гіпертензія; псевдорезистентна артеріальна гіпертензія; клінікофункціональна характеристика.

\section{КЛИНИКО-ФУНКЦИОНАЛЬНАЯ ХАРАКТЕРИСТИКА РЕЗИСТЕНТНОЙ АРТЕРИАЛЬНОЙ ГИПЕРТЕНЗИИ}

○С. В. Бусел

\section{Одесский национальный медицинский университет}

РЕЗЮМЕ. Цель исследования - изучить клинико-функциональные особенности резистентной и псевдорезистентной артериальной гипертензии. 
Огляди літератури, оригінальні дослідження, погляд на проблему

Материал и методы. Было обследовано 420 больных с эссенциальной артериальной гипертензией 2 стадии в возрасте от 45 до 74 лет и продолжительностью заболевания более 3 лет с признаками резистентности к проводимой гипотензивной терапии. Все пациенты были поделены на 2 группы: 1 группа - с резистентной АГ и 2 группа - с псевдорезистентной АГ. У всех пациентов оценивали данные анамнеза, жалобы, продолжительность заболевания АГ, наличие сопутствующих заболеваний. Были проведены все необходимые общеклинические и инструментальные обследования соответственно поставленным задачам исследования: общеклиническое исследование, ЭКГ, ЭхоКГ.

Результаты. В ходе исследования у 26,2 \% пациентов была диагностирована истинная резистентная АГ, у 73,8 \% - псевдорезистентная АГ. Длительность псевдорезистентной АГ в среднем составила $(8,2 \pm 1,2)$ лет, а резистентной АГ - почти вдвое больше - $(14,7 \pm 2,1)$ лет. У больных с резистентной АГ по сравнению с больными с псевдорезистентной АГ было выявлено больше жалоб, более высокие значения систолического артериального давления, чаще встречались сопутствующая патология и нарушения сердечного ритма и проводимости.

Выводы. У больных с резистентной АГ отмечается более тяжелое течение АГ, что создает условия для повышения общего кардиоваскулярного риска.

КЛючЕВЫЕ СЛОВА: резистентная артериальная гипертензия; псевдорезистентная артериальная гипертензия; клинико-функциональная характеристика. 